\title{
Multiway Partial Least Square (MPLS) to estimate impact localization in structures
}

\author{
L.E. Mujica* M. Ruiz ${ }^{* *}$ X. Berjaga ${ }^{* *}$ J. Rodellar ${ }^{* * *}$ \\ * Department of Applied Mathematics III. Escola Universitària \\ d'Enginyeria Tècnica Industrial de Barcelona (EUETIB). Universitat \\ Politécnica de Catalunya (UPC). Comte d'Urgell 187, 08036, \\ Barcelona, Spain. \\ e-mail: luis.eduardo.mujica@upc.edu \\ ** Department of Electrical, Electronic and Automatic Control \\ Engineering. Institut d'Informàtica $i$ Aplicacions (IIiA). University of \\ Girona. Campus Montilivi, Edifici P-IV, 17071. Girona, Spain. \\ e-mail: $\{$ mlruizo, xberjaga\}@eia.udg.edu \\ *** Department of Applied Mathematics III. Escola Tècnica Superior \\ d'Enginyers de Camins, Canals i Ports de Barcelona (ETSECCPB). \\ Universitat Politécnica de Catalunya (UPC). Jordi Girona, 1-3, \\ 08034, Barcelona, Spain. \\ e-mail: jose.rodellar@upc.edu
}

\begin{abstract}
This paper presents results from the application of Multiway Partial Least Square (MPLS) as a regressor tool in order to estimate the localization of impacts in an aircraft structure. MPLS is a technique that maximizes the covariance between the predictor matrix $X$ and the predicted matrix $Y$ for each component of the space. The structure can be considered as a small scale version of part of a wing aircraft. 574 experiments were performed impacting the wing over its surface and receiving vibration signals from nine sensors. Experiments are divided in four groups depending on their localization and probability of occurrence. A PLS model is build using three of these groups and tested using the remaining group. Results are presented, discussed and compared with results of other methods.
\end{abstract}

\section{INTRODUCTION}

\section{IMPACT DAMAGE IDENTIFICATION IN STRUCTURES}

\subsection{Overview}

Having the capability for detecting damages on structures in the incipient state is one of the greet challenges in mechanical, aerospace and aeronautical industry, among others. On the one hand, this will guarantee the integrity of the structure, increasing the security. On the other hand, maintenance and repairing costs could be considerably reduced.

In the last years, different techniques under the concept of "Structural Health Monitoring (SHM)" have continuously been in development. Recently, Worden and Farrar [2007] have compiled an especial issue on SHM, introducing its concept, an overview about sensing systems, inverse methods, time and frequency scale methods. Staszewski et al. [2004] in their multidisciplinary book cover all recent developments in smart sensor technology for health monitoring in aerospace structures, providing a valuable introduction to damage detection techniques.

Damages due to an external impact are a major concern in the design of aerospace structures. For instance, low 
velocity impacts can cause delamination in composite materials. Impacts can occur during manufacture, service or maintenance and the main causes are: fall of tools, collision with animals, runway stones, debris, or ballistic impacts.

In many cases, the identification of this damage (known as Barely Visible Impact Damage -BVID-) by using visual inspections is difficult. In structures which are susceptible to impacts, NDT (Non Destructive Testing) routines must be performed over the entire surface since the location of the impact is unknown. These is a very time consuming task that entiles a high economic cost and requires that the structure is out of service. Therefore, systems that can detect the occurrence of impacts and estimate its location and its energy are very helpful in structural maintenance (Choi and Chang [1996]).

\subsection{Principle of impact identification}

If a structure is exposed to a mechanical impact on the surface, a transient strain pulse is introduced. The acoustic or strain wave is propagated in the structure along spherical wavefronts. The arrival of these waves at the surface where the impact was generated produces displacements which are measured by means of receiving transducers or sensors. There exist in the literature a considerable number of studies on acoustic waves generated by impact sources in metallic and composite materials (e.g. Gardiner and Pearson [1985], Weems et al. [1991], Takeda et al. [1981] and Prosser et al. [1999].

Once, the structural area is equipped with a set of sensors, at least two different evaluations should be performed: (i) Impact identification, simultaneously with the impact occurrence, and (ii) Impact damage identification, or analysis of post-impact damage. The former is focused on the detection, location and, in some cases, determination of the impact force, by using a passive system. The latter usually uses an active system to assess the structure after the impact. This assessment includes whether damage is produced, its location and severity.

\subsection{Impact identification as prediction problem}

Approaches developed until now for impact location and identification can be classified in two main groups: Analytical Model Based and Data-Driven Model Based. The former uses a first principle models obtained from physical laws. Some of them are based on equations of motion which characterizes the dynamic response of the structure subject to known impact. The latter main group is based on data driven models. These models are able to describe complex relationships between input and output data when specific equations are not applicable. A considerable amount of time domain data is needed. Mujica et al. [2009] presents and extensive review of methodologies for impact damage detection in structures using strain data.

Within Data-Driven Model Based concept, methodologies based on classification, clustering, pattern recognition and regression have been proposed. Classifiers determine a zone of the structure where the impact were produced. On the other hand, regressors try to estimate or predict an exact position of the impact. Artificial Neural Networks
(ANN's) are the most important and widely used predictor in SHM. Worden and Staszewski [2000] and Staszewski et al. [2000] used two ANN's to predict impact location and energy in composite materials. Typical multilayer perceptrons were trained with experimental data using the backpropagation learning rule. This approach was tested by Haywood et al. [2005] in Smart Layer and by LeClerc et al. [2007] in a large aircraft structure.

\section{MULTIWAY PARTIAL LEAST SQUARE (MPLS)}

MPLS is a technique that maximizes the covariance between the predictor matrix $X$ and the predicted matrix $Y$ for each component of the space (Russell et al. [2000]). The data is stored in a three-dimensional (3D) $\underline{X}$ matrix. This matrix is organized by $I$ Experiments, $K$ Time instants and $J$ Sensors. Afterwards, the $\underline{X}$ matrix is unfolded in a two dimensional (2D) $X$ matrix in direction of the experiments as is shown in 1 . Where each frontal slice represents all measurements from one sensor. Each front slice is put next to each other, therefore one row represents the data from one experiment. When the $X$ matrix is ready, the data is scaled and the PLS algorithm is applied.

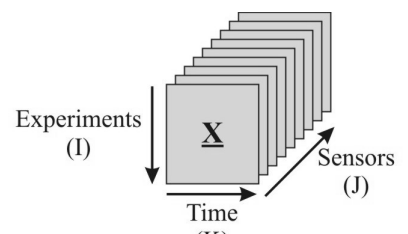

(K)

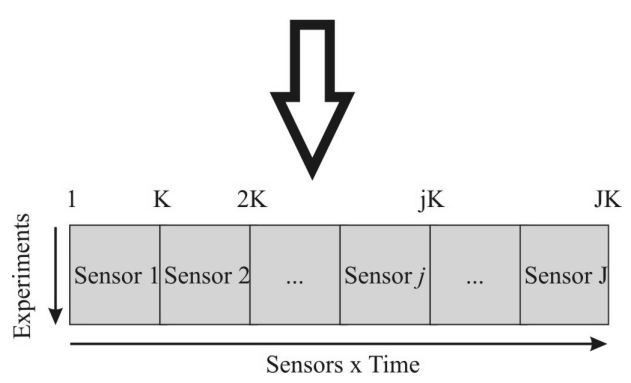

Fig. 1. Predictor Matrix (X): Unfolding from 3D to 2D

The predicted matrix $Y \epsilon R^{n \times m}$ contains the impact location as can be seen in Figure 2.

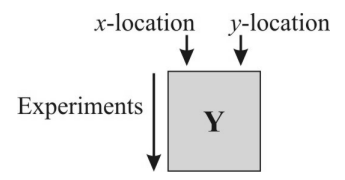

Fig. 2. Predicted Matrix (Y)

PLS requires calibration and prediction steps. The goal of PLS is to determine the loading and score vectors which are correlated with $Y$ while describing a large amount of the variation in $X$. The PLS is achieved by decomposing $X$ and $Y$ into a combination of loadings $P$ and $Q$ (these are determined by orthogonal vectors), scores $T$ (the projection of the loading vectors associated with the first singular values) and residual matrices $E$ and $F$ (Kourti [2002]).

$$
X=T P^{T}+E
$$




$$
Y=T Q^{T}+F
$$

The matrix product $T P^{T}$ can be expressed as the sum of the product of the score vectors $t_{j}$ (the $j^{\text {th }}$ column of $T$ ) and loading vectors $p_{j}$ (the $j^{\text {th }}$ column of $P$ ). Similarly, $Y$ is decomposed as the sum of the product of the score vectors $t_{j}$ (the $j^{\text {th }}$ column of $T$ ) and loading vectors $q_{j}$ (the $j^{\text {th }}$ column of $Q$ ) (Russell et al. [2000]).

$$
\begin{aligned}
& X=\sum_{j=1}^{N} t_{j} p_{j}^{T}+E \\
& Y=\sum_{j=1}^{N} t_{j} q^{T_{j}}+F
\end{aligned}
$$

where $N$ is the number of principal components deemed to be significant.

\section{EXPERIMENTAL SETUP}

This section describes the same experimental setup previously used to demosntrate that the problem of identifying impact locations can be solved successfully using several approaches, for instance: Artificial Neural Networks can be used as a regressor, a classifier and as a combination of both (LeClerc et al. [2007]). Another option is the utilisation of Genetic Algorithms (Mahzan et al. [2007]) or Case Based Reasoning combined with different dimensionality reduction (or features extraction) techniques sucha as: Self Organizing Maps -SOM- (Mujica et al. [2008]), Principal Component Analysis (PCA), Partial Least Square (PLS), Curvilinear Distance Analysis (CDA) and some extensions of them (Mujica et al. [2008]).

The structure used in this work is a section of a commercial aircraft wing flap with the corresponding leading and trailing edges as shown in Figure 3.

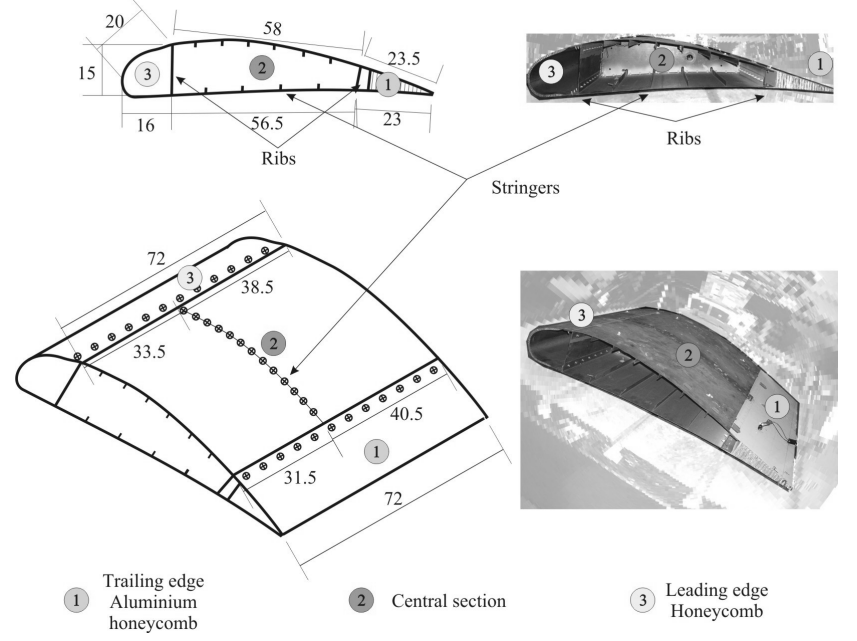

Fig. 3. Wing flap section. (Dimensions in $\mathrm{cm}$.)

To impart strength into important areas of the panel, there exist various ribs, spars and stringers running throughout the structure, as well as the use of honeycomb cores at the leading and trailing edges. The trailing edge is composed of aluminium skins with an aluminium honeycomb core, the leading edge of composite skins with a light weight honeycomb core and the central section of thin composite material. Unfortunately, due to the nature of the origin of the wing flap section (being from a commercial aircraft) little is known about the specific materials and design parameters.

Nine low-profile, surface-bounded piezoceramic sensors (PIC 155, 10mm in diameter and $1 \mathrm{~mm}$ thick) are used to measure the impact strain data. Sensors and connectors are distributed over the surface of the flap: two on the leading edge, two on the trailing edge and five in the central section as shown in figure 4a.

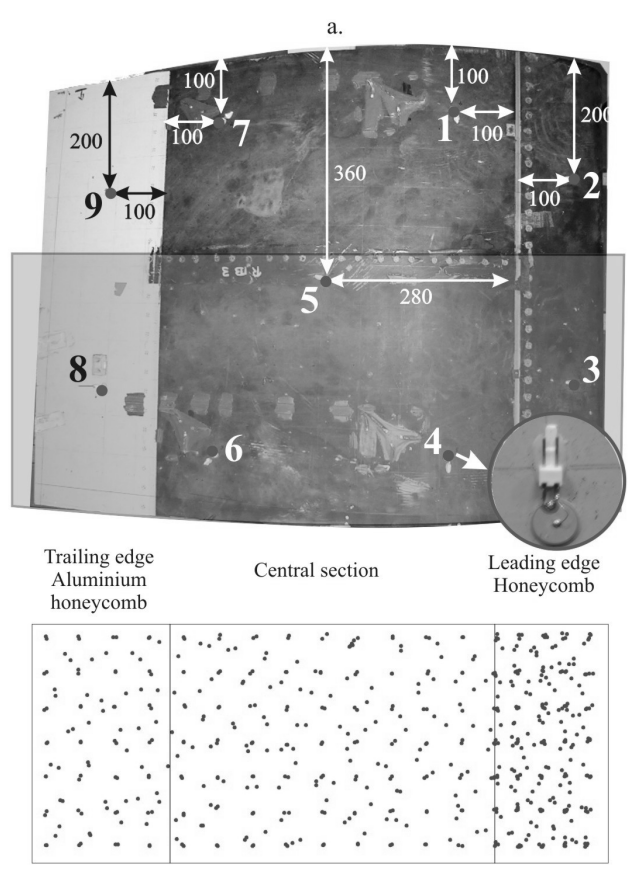

b.

Fig. 4. Wing panel: (a) Sensor locations and superimposed impacted zone, and (b)Impact locations measured.

The surface test area shown in figure $4 \mathrm{a}$, is impacted using a rubber tipped PCB instrumented hammer that is chalked before impact to mark the exact position of the impacts. Due to the importance of the leading edge with regards a higher probability of impacts during flight from sources such as bird strikes, more impacts are repeated on the leading edge. The response from all nine sensors on the structure are recorded for a total of 574 impacts, whose positions are measured from the $x$ and $y$ datum lines. The distribution of the measured impacts over the test area can be seen in Figure 4b.

Signals obtained during testing contain a lot of undesirable information, including: noise, different levels of unavoidable offset, trends, large amount of data-points,etc. (see figure 5a). Offsets are removed using the mean value of each signal. Furthermore, signals are cut off, eliminating data-points which does not contain any information (see Figure $5 \mathrm{~b}$ ). Finally, the set of these signals is arranged in a $3 D$ matrix, where $j=1,2, \ldots, J$ sensors are recorded at $k=1,2, \ldots, K$ time instants throughout a particular experiment. Similar data are generated for a number of such experiment runs $i=1,2, \ldots, I$. That generates a 
three-way data array $\underline{X} \epsilon \mathbb{R}^{I x J x K}$ as it was illustrated in Figure 1, where height gives the number of experiments $I$, width gives the number of time instants $K$, and length gives the number of measurements (sensors) $J$. In this way, each frontal slice is a $2 D$-matrix $X$ which represents all measurements in one sensor. On the other hand, the position of the impact ( $x$-location and $y$-location) is stored in a matrix $Y$ as can be seen in Figure 2 .

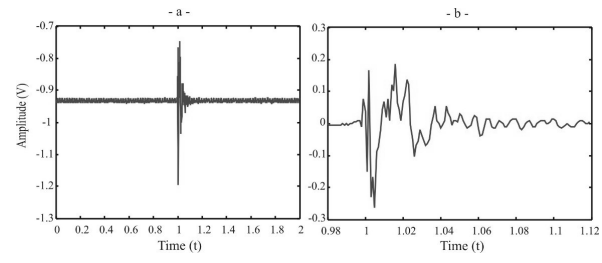

Fig. 5. Signal pre-processing example. (a) Sensed signal, (b) Final signal

\section{IMPACT LOCATION METHODOLOGY}

\subsection{Overview}

This section describes a methodology for impact location using MPLS. Accuracy of this methodology is assessed using cross validation. Training data is used to built a PLS model, afterwards, using the testing data, the localization of impacts is predicted. A radial error between real position and predicted position is calculated.

\subsection{Cross Validation}

A fundamental issue is to assure that results represent any data distribution. The model has to cover all the working area where the experiments have been performed. To solve this situation, the cross validation algorithm is used. The goal of this algorithm is to divide into several sets of data of approximately the same number of experiments. The remaining data is used for the validation of the model.

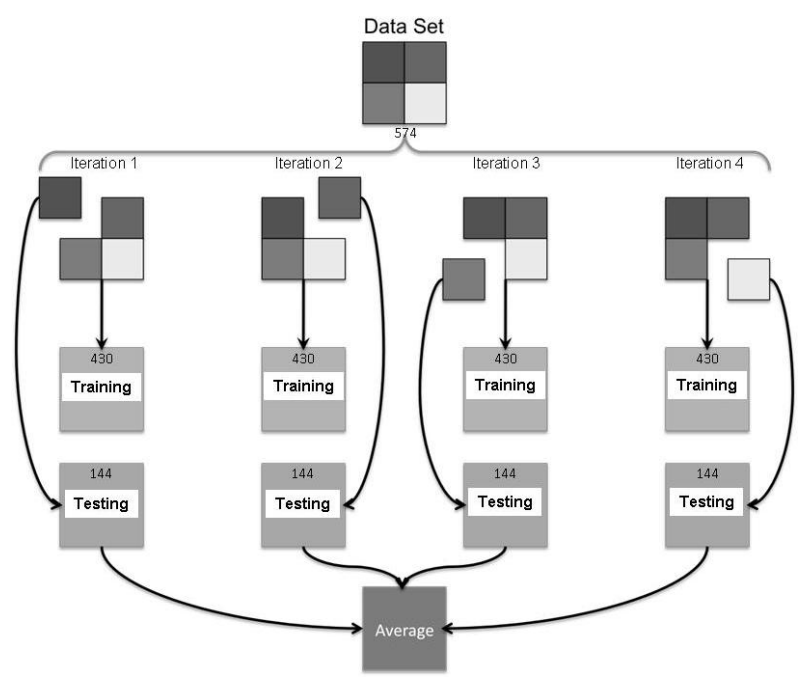

Fig. 6. Cross Validation procedure

In this work, the totality of experiments (574) have been divided in four sets, guaranteeing several impact location in each one. The procedure of this algorithm is presented in Figure 6.

\subsection{PLS as predictor}

Once, the data is divide in sets, the methodology developed to use MPLS as a predictor is applied. This methodology is organized in three steps as shown in Figure 7), and is applied four times (four iterations), once for each data set given by the cross validation algorithm.

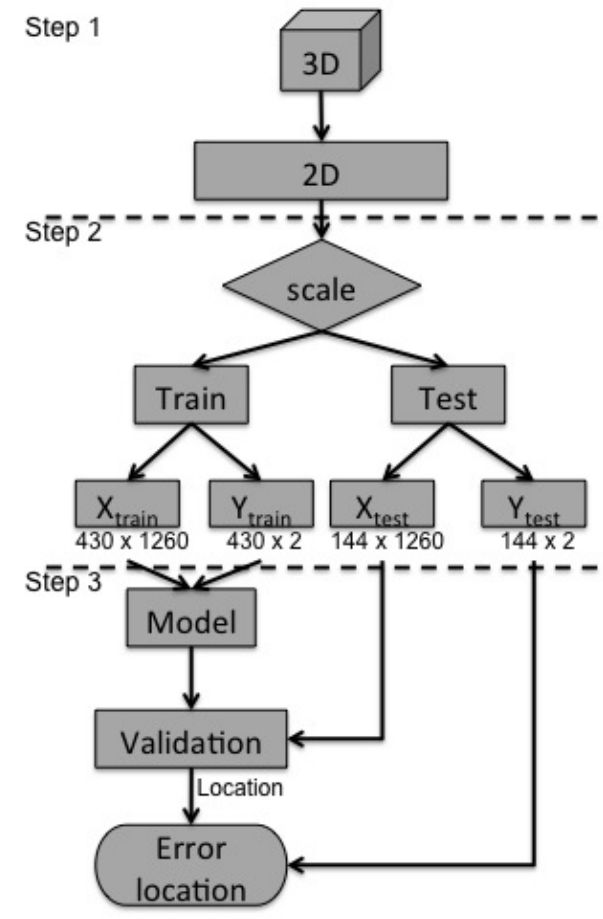

Fig. 7. MPLS Methodology developed for prediction of impact location

Step 1. Data organization (Multiway): The $3 D$ matrix $\underline{X}$ collected by experiments is unfolded in the direction of the experiments where all the sensors are related with every time instant into matrix $X$. This matrix $X$ contains vibration signals from all sensors for each impact. The matrix $Y$ has the coordinates of the impact for each experiment.

Step 2. Data division and scaling: Once the array of signals is given in two dimensions, data is divided in two parts: the first one is used for building the model (training) and the second one for validation (testing). These two matrices are related to their respective impact location (Matrix $Y$ ). Then, those matrices are scaled and PLS can be applied. In this work, the main idea of using PLS is to extract the information contained by the combination of sensors through time to predict the position of impact.

Step 3. PLS application: The model is built using the training matrices $X_{\text {train }}(430 \times 1260)$ and $Y_{\text {train }}(430 \times 2)$, where 430 correspond to the total number of experiments, 1260 is the combination of the 9 sensors in 140 time instants and 2 correspond to the actual impact location ( $x$-location, $y$-location). To predict the impact location of 144 experiments, $X_{\text {test }}$ matrix $(144 \times 1260)$ is projected into the model. Finally, to determine the accuracy of the methodology, predicted locations are compared with the real location of these impacts ( $Y_{\text {test }}$ matrix). 


\section{ANALYSIS RESULTS AND DISCUSSION}

In order to compare predicted locations with real locations, radial errors are calculated as follow:

$$
\text { radial }_{\text {error }}=\sqrt{\left(x_{\text {real }}-x_{\text {pred }}\right)^{2}+\left(y_{\text {real }}-y_{\text {pred }}\right)^{2}}
$$

Using 41 principal components, the percent variance captured in $X$ and $Y$ and the average radial error by iteration are shown in Table 1. Finally, the total average radial error is around $60 \mathrm{~mm}$.

\begin{tabular}{|c|c|c|c|}
\hline & $\begin{array}{c}\text { Percent Variance } \\
\text { Captured in X }\end{array}$ & $\begin{array}{c}\text { Percent Variance } \\
\text { Captured in Y }\end{array}$ & $\begin{array}{c}\text { Average Radial } \\
\text { error }(\mathrm{mm})\end{array}$ \\
\hline It. 1 & 92.71 & 98.37 & 62.21 \\
\hline It. 2 & 92.62 & 98.44 & 60.57 \\
\hline It. 3 & 92.50 & 98.52 & 59.69 \\
\hline It. 4 & 92.41 & 98.59 & 59.06 \\
\hline \hline Total & 92.56 & 98.48 & 60.38 \\
\hline
\end{tabular}

Table 1. Total variance captured and average radial error per iterations

Radial error in millimeters by experiment per each iteration can be seen from Figures 8 to 11 .

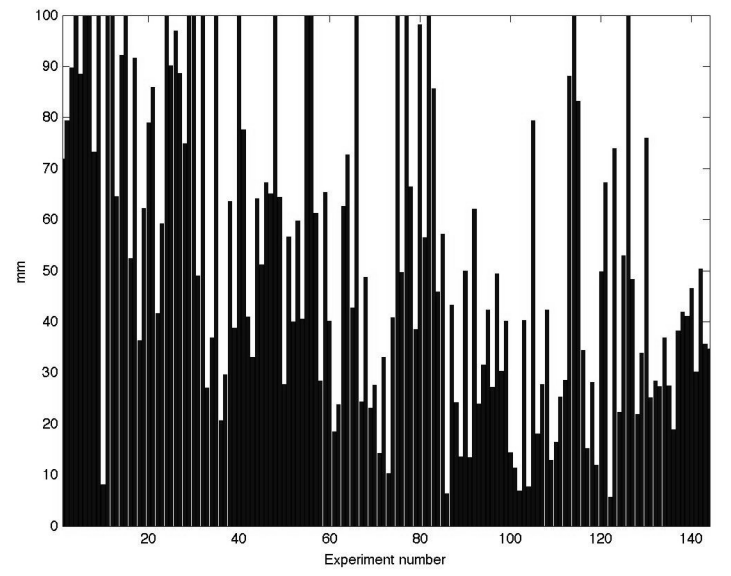

Fig. 8. Error for each experiment in iteration 1

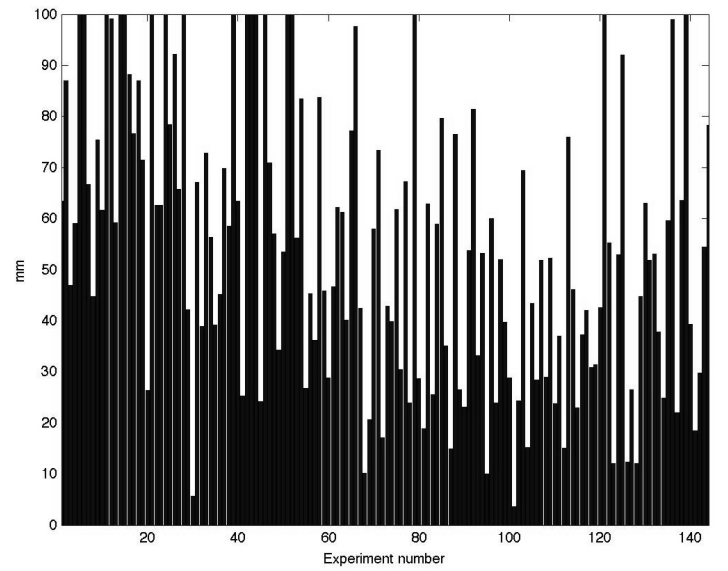

Fig. 9. Error for each experiment in iteration 2

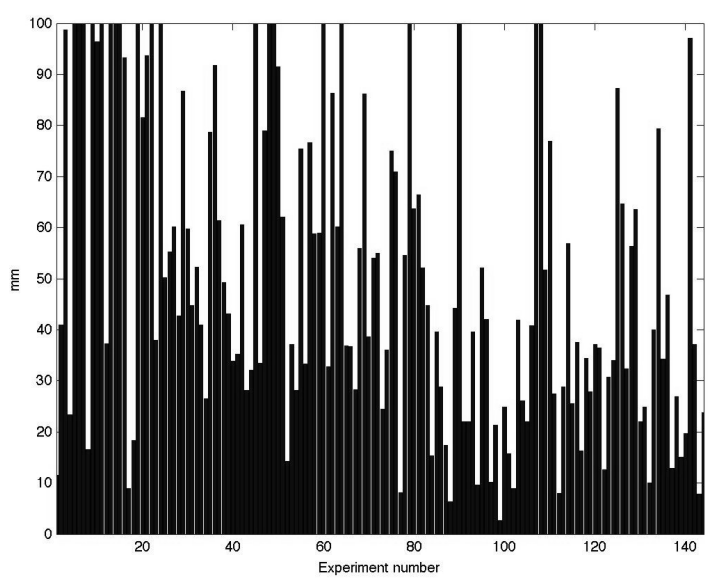

Fig. 10. Error for each experiment in iteration 3

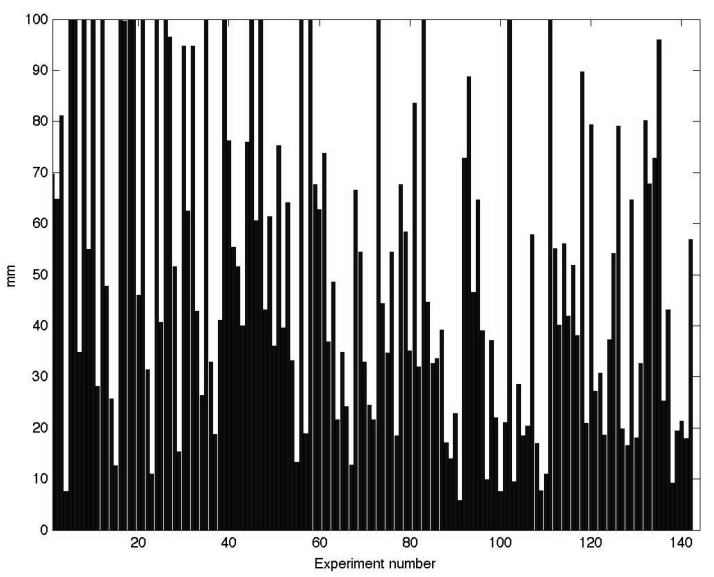

Fig. 11. Error for each experiment in iteration 4

Results obtained in this work are similar to the ones presented in previous works that used different techniques such as Artificial Neural Networks (LeClerc et al. [2007]), Genetic Algorithms (Mahzan et al. [2007]) and Case Based Reasoning (Mujica et al. [2008])(Mujica et al. [2008]). However, the methodology presented in this work uses the information gathered from the sensors, without computing new or additional features. Moreover, it can be seen that it is not necessary to combine this methodology with another procedure to obtain acceptable results. Additionally, it is well known that Artificial Neural Networks and Genetic Algorithms require previous and deep knowledge of the experiment in order to configure them and set the initial parameters, whereas MPLS only requires of historical data of the events.

\section{CONCLUSION}

In this work, the unique tool used was Multiway Partial Least Square (MPLS). This technique has been applied to reduce the dimensionality of the problem, and besides to predict or estimate the location of new impact on aircraft structures. Data was previously organized in several sets in order to guarantee that data choose to built the model and test it are distributed over all the surface of the structure. 
This set of data is stored in a 3D matrix which is unfolded to get a 2D matrix with 544 experiments and 1260 samples $x$ time.

After applying the PLS algorithm, the dimension samples $x$ time is reduced from 1260 to 41 . In each iteration, the model is built using also the locations of the impact (supervised training). When the testing data is projected into the model, a new matrix $Y$ is calculated, which contains the predicted location of each impact.

Results show the high degree of accuracy to locate impact in structures. Further work will be focused in estimating the fault magnitude of the impact by means of the methodology here presented.

\section{ACKNOWLEDGEMENTS}

The authors thank the Ministerio de Ciencia e Innovación in Spain through the research project DPI2008-06564C02-02 and the young post-doctoral programme "Juan de la Cierva". We are grateful also to Ms Jenny LeClerc, Wieslaw Staszewski and Keith Worden from University of Sheffield who have collected the data and contributed with valuable information.

\section{REFERENCES}

P.T. Coverley and W.J. Staszewski. Impact damage location in composite structures using optimized sensor triangulation procedure. Smart Materials and Structures, 12:795-803, 2003.

J. Haywood, P.T. Coverley, W.J. Staszewski, and K. Worden. An automatic impact monitor for a composite panel employing smart sensor technology. Smart $M a-$ terials and Structures, 14:265-271, 2005.

R.T. Jones, J.S. Sirkis, E.J. Friebele and A.D. Kersey. Location and magnitude of impact detection in composite plates using neural networks. In William B. Spillman, editor, Proc. SPIE Vol. 2444, Smart Structures and Materials 1995: Smart Sensing, Processing, and Instrumentation, pages 469-480, 1995.

L.E. Mujica, J. Vehí, W.J. Staszewski, and K. Worden. Impact damage detection in aircraft composites using knowledge-based reasoning. Structural Health Monitoring, an International Journal, 7(3):215-229, 2008.

W.J. Staszewski. Monitoring on-line integrated technologies for operational reliability - monitor. Air 83 Space Europe, 2(4):67-72, 2001.

W.J. Staszewski, K. Worden, R. Wardle, and G.R. Tomlinson. Fail-safe sensor distributions for impact detection in composite materials. Smart Materials and Structures, 9:298-303, 2000.

K. Worden and W.J. Staszewski. Impact location and quantification on a composite panel using neural networks and a genetic algorithm. Strain, (36):61-70, 2000.

J. LeClerc, K. Worden, W.Satszewski, and J. Haywood. Impact detection in an aircraft composite panel - a neural-network approach. Journal of Sound and Vibration, 299:672-682, 2007.

S. Mahzan, W.J. Staszewski, and Worden K.: Comparative study of impact damage detection in aerospace structures. In Mahzan et al. [2007], pages In CD-ROM.

L.E. Mujica, J. Vehí, M. Ruiz, M. Verleysen, W. Staszewski, and K. Worden. Multivariate statistics process control for dimensionality reduction in structural assessment. Mechanical Systems and Signal Processing, 22:155-171, 2008a.

K. Choi and F.K. Chang. Identification of impact force and location using distributed sensors. AIAA journal, 34(1):136-142, 1996.

D.S. Gardiner and L.H. Pearson. Acoustic-emission monitoring of composite damage occurring under static and impact loading. Experimental Techniques, 9:22-28, 1985.

W.H. Prosser, M.R. Gorman, and D.H. Humes. Acoustic emission signals in thin plates produced by impact damage. Journal of Acoustic Emission, 17(1-2):29-36, 1999.

W.J. Staszewski, C. Boller, and G.R. Tomlinson. Health Monitoring of Aerospace Structures: Smart Sensor Technologies and Signal Processing. John Wiley \& Sons, Ltd, Chichester, 2004.

N. Takeda, R.L. Sierakowski, and L.E. Malvern. Wave propagation experiments on ballistically impacted composite laminates. Journal of Composite Materials, 15: 157-175, 1981.

D. Weems, H.T. Hahn, E. Granlund, and I.G. Kim. Impact detection in composite skin panels using piezoelectric sensors. In In American Helicopter Society 47th Annual Forum Proceedings, pages 643-652, 1991.

K. Worden and C.R. Farrar. Theme issue: Structural health monitoring. Philosophical Transactions of the Royal Society, Series A, 365(1861):299-632, 2007.

L.E. Mujica, J. Rodellar, and J. Vehí. A review of impact damage detection in structures using strain data. International Journal of COMADEM, In printing, 2009.

Theodora Kourti. Process analysis and abnormal situation detection: From theory to practice. IEEE Control Systems Magazine, 22(5):10-25, oct 2002.

Evan L. Russell, Leo H. Chiang, and Richard D. Braatz. Data-driven techniques for fault detection and diagnosis in chemical processes "Advances in Industrial Control". ISBN 1-85233-258-1, London, 2000.

Svante Wold, Paul Geladi, Kim Esbensen, and Jerker Ohman. Multiway pincipal component and pls analysis. Journal of Chemometrics, 1:41-56, 1987. 